\title{
DESign AND Simulation ANALYSIS OF HAPTIC DEVICE With PARAllel Kinematics
}

\author{
Elena Kuznetcova*, Victor Titov, Evgeniy Smirnov, \\ Igor Dalyaev and Alexander Truts
}
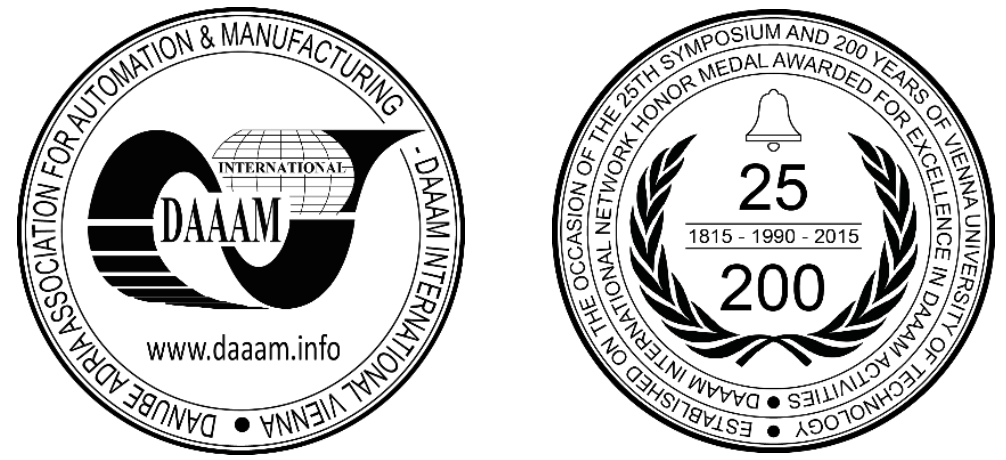

This Publication has to be referred as: Kuznetcova, E[lena]; Titov, V[iktor]; Smirnov, E[vgeniy]; Dalyaev, I[gor] \& Truts, A[lexander] (2018). Design and Simulation Analysis of Haptic Device With Parallel Kinematics, Proceedings of the 29th DAAAM International Symposium, pp.0636-0644, B. Katalinic (Ed.), Published by DAAAM International, ISBN 978-3-902734-20-4, ISSN 1726-9679, Vienna, Austria

DOI: $10.2507 / 29$ th.daaam.proceedings.092

\begin{abstract}
The paper presents a description of a haptic device design for remote control of robotic systems. The device includes actuators with torque motors utilized in force feedback for a human operator. Partial mechanical compensation of gravity torques is employed to facilitate the actuation, reduce the motors size and consumption. The kinematic workspace and accuracy map of the device is calculated using Monte Carlo simulation to verify the achievement of the design objectives. As a main output signals position/orientation, velocity and accelerations are chosen as the most applicable for general motion specification. An advanced method utilizing Kalman filter approach for estimation of velocity and acceleration from positions measurements is presented along with simulation results.
\end{abstract}

Keywords: haptic device; parallel kinematics; Kalman filter; velocity/acceleration estimation

\section{Introduction and motivation}

The manual control of robotic systems has been an acute topic for a several decades as it was probably the first types of control applied in the field. As the capabilities of the robotics systems in terms of control and high level reasoning grow the necessity for remote manual control stays relevant since the amount of tasks that still hardly budge to automation (like complex manipulation in undetermined environment with limited or uncertain feedback information) remains almost stable. Primarily these tasks arise in the field of "extreme robotics" covering emergency recovery, extreme environment (radiation, chemicals, explosives, etc) and healthcare (medical industry). A somewhat unique field requiring (almost always mandatory) manual control is outer space servicing operations (spacecraft repair/modification, space debris collection, satellite maintaining, etc).

The common problems for the areas are

- complex (often) undetermined environment;

- requirement for precise manipulation;

- $\quad$ lack (or bad quality) of appropriate feedback;

- high risks and cost of mistakes. 
Automation of such tasks is far from being trivial while human operator can cope with them relatively easy given the necessary minimum of information (such is the typical situation for teleoperation). In particular teleoperation tasks the information about contact is useful an operator. That feedback is provided in haptic devices which employed in creation of bilateral control systems.

Even when a human is just a supervisor for autonomous task execution he/she still needs the means to specified the task and/or correct the results of planning. An operator has different means of specifying the motion of the executing mechanism which can roughly be divided into two categories: specifying the motion task in the task space (can be facilitated by high level machine reasoning and planning) with software GUI (and/or virtual reality) or the direct teleoperation with an input device. Regardless of the category the minimal output information from input devices is position/orientation but in order to pass dynamic characteristics of motion from an operator to the control system the derivatives of the signals are needed.

Haptic devices used for teleoperation differ in the number of degrees of freedom (DoF) ranging from a classical 2D joystick to a full kinematic copy of the executive (controlled) device. The later requires much space and physical activity from an operator but gives most accurate and representative for manual remote control [1]. The scaled but kinematically equivalent (similar) haptics (like "МЭМ-10СДГ" [1] or Kraft mini master [2], fig. 1) are more convenient in this sense and well suited for ad-hoc applications.

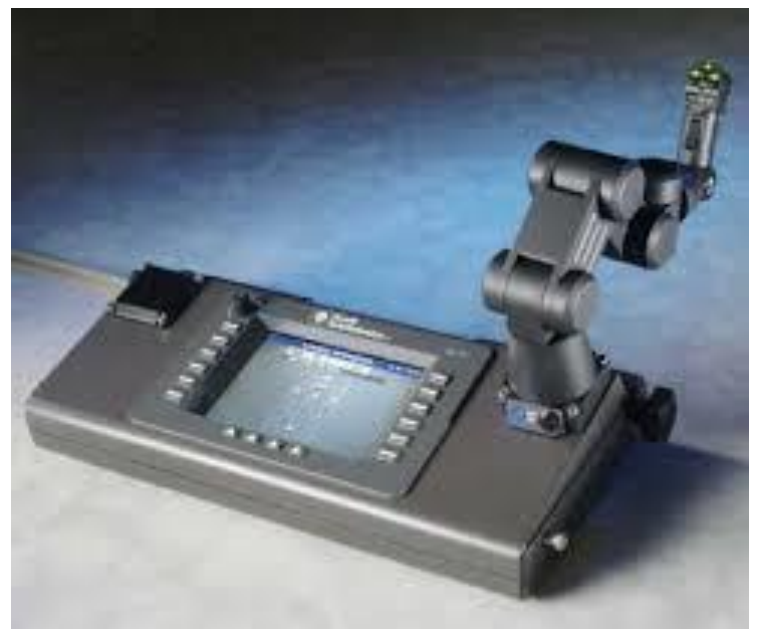

Fig. 1. Kinematical similar haptic example: Kraft Force Feedback Mini-Master

For most cases kinematic similarity is not a requirement while the functionality to uniquely specify the pose of a manipulator end-effector (and/or dynamic properties of motion) is what actually needed.This paper presents a universal haptic device and a signal processing approach to estimate up to second order derivative of the position signals. The design for the universal haptic device suitable for a wide range of application and exploitation conditions is presented. The force feedback approach implemented in the device incorporate the results previously presented in [3].

The rest of the paper organized as follows. First the design objectives are formulated and explained in section 2 . The mechanical design and evaluation is described in following section 3. A method for velocity and acceleration estimation is proposed in section 4 and tested in simulation with results presented in section 5 .

\section{Mechanical design and evaluation}

This section is devoted to the mechanical design description of the proposed haptic device.

The parallel kinematic structure base on the Claves delta mechanism is utilized. The parallel mechanism is preferred due to its attractive mechanical properties [4]: low inertia, high stiffness, low friction, backdriveability, near-zero backlash (or possibility to remove them), gravitationnal counterbalancing, sizeable workspace, multiple degrees-of-freedom, and human matched force capabilities. The parallel kinematics is used to specify three Cartesian coordinate as it allows only linear motion of the handle. The orientation measurements are provided by additional three degrees of freedom. The closest to the device in this paper is Sigma7 haptic device [5] which kinematic structure has been repeated.

The central moving platform can take only linear displacements and keeps its orientation relative to the base platform. All the actuated joints (1-6, figure 2) are direct drive type (no gear boxes) that helps to increase the efficiency of the torque transfer and mitigate the torque pulsation that occurs in harmonic drives or the backlashes in the planetary gears. This also enhance the dynamic response of the system as well as allowing direct force control (or compliancy control) that requires no torque sensors in the joints to produce the required force at the end-effector. Each actuator contains a brushless DC torque motor and a precise optical absolute encoder type position sensor. For partial compensation of gravity torques a spring system tuned to a single set position is applied. The springs stiffnesses are chosen to minimize the parasitic influence occurring when system is moved out of the set position. 


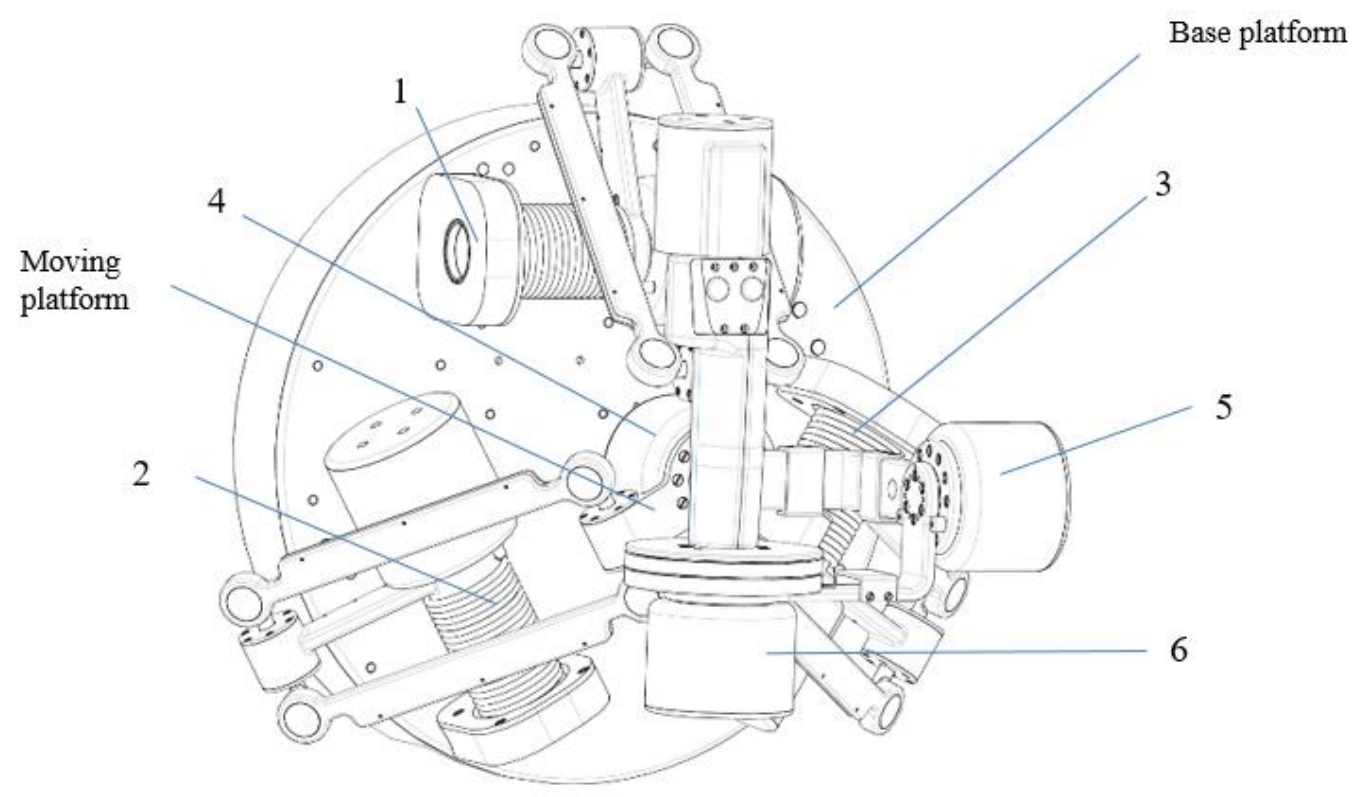

Fig. 2. Haptic device with parallel kinematics

The links geometry was optimized to fit the required workspace: the spherical sector of $280 \mathrm{~mm}$ in radius and the height of 130. The orientaional DoFs are to cover rotation about $\mathrm{X}, \mathrm{Y}, \mathrm{Z}$ axes by $\pm 90^{\circ}$ (covers the range of the physiologically consistent angles of the human wrist with the forearm being fixed). After the optimization and taking into account some engineering considerations the actual workspace was obtained in Mote Carlo simulation (figure 3). It fully covers the required workspace with minimal overshoot.

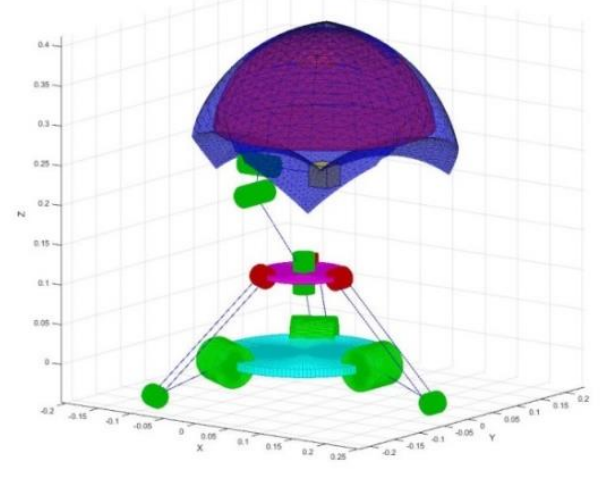

Fig. 3. Actual workspace

In the same Mote Carlo simulation the pose measurement errors were estimated. The accuracy of the optical encoder $( \pm 150 ")$ was used for the estimation. In figure 4 the positioning errors map due to the parallel mechanism is depicted in the three planes. Table 1 contains numerical values of the errors. Figure 5 shows the distributions of the errors.
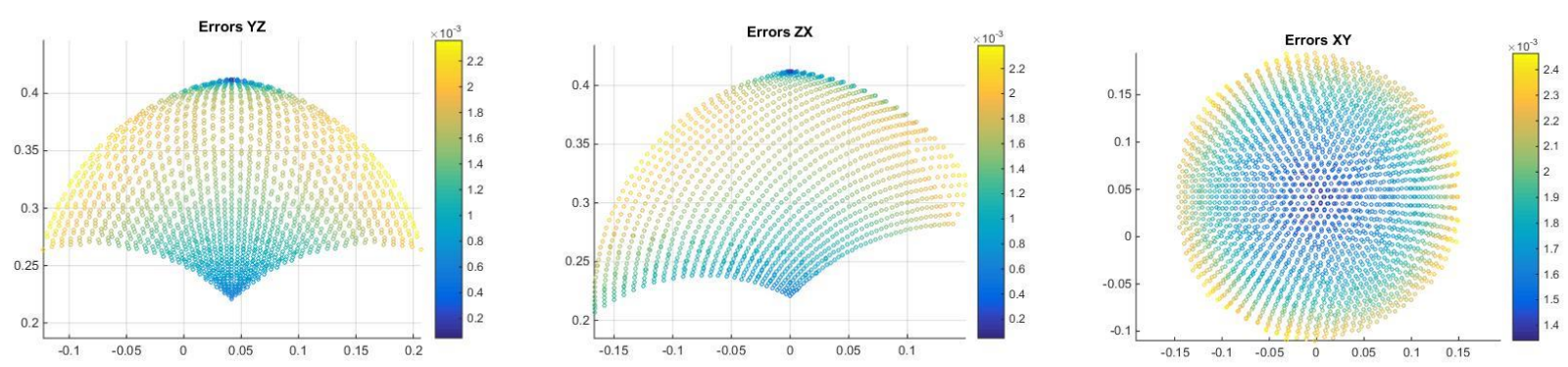

Fig. 4. Linear position measurement error 


\begin{tabular}{|c|c|c|c|}
\hline & $\mathbf{X}$ & $\mathbf{Y}$ & $\mathbf{Z}$ \\
\hline Max. value of abs. err., $\mathrm{mm}$ & 2.2 & 2.2 & 1.6 \\
\hline Mean value of abs. err., $\mathrm{mm}$ & 1.4 & 1.3 & 0.9 \\
\hline STD of abs. err., mm & 0.3 & 0.2 & 0.3 \\
\hline $\begin{array}{c}\mathrm{P} 4 \\
\end{array}$ & 4 & $\mathrm{~s}$ & \\
\hline Max. norm of err. vector, mm & \multicolumn{3}{|c|}{2.5} \\
\hline Mean norm of err. vector, $\mathrm{mm}$ & \multicolumn{3}{|c|}{1.8} \\
\hline STD of err. vector, $\mathrm{mm}$ & \multicolumn{3}{|c|}{0.3} \\
\hline Orientation accuracy & \multicolumn{3}{|c|}{ $\pm 450 "$} \\
\hline
\end{tabular}

Table 1. Measurement error statistics
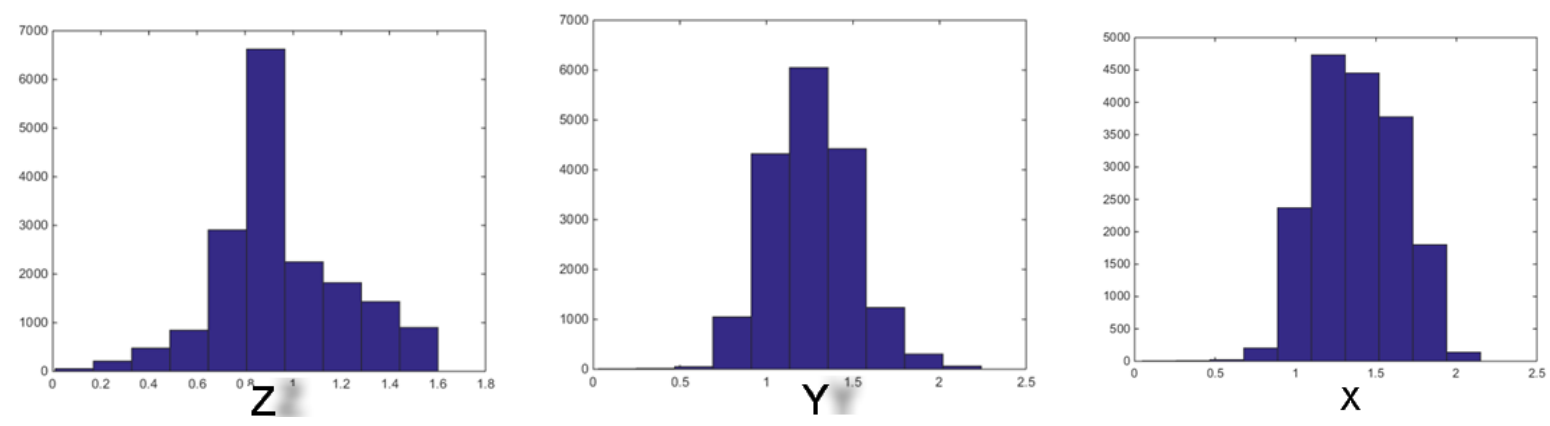

Fig. 5. Linear position measurement error distribution

\section{Measurements and signal processing}

A haptic device is supposed to provide commanding signals to control system of the executive manipulator. This signals can be obtained after appropriate transformation from the built-in position sensors and the 6 DoF force/torque sensor (FTS). Due to the high stiffness of the FTS (for the forces produced by a human-operator) and low inertia of the handle the signals for the FTS can be used directly with regular noise filtering techniques if required.

The measurements of the angular position sensors built in the actuators are used to calculate the Cartesian pose of the handle that is enough to specify the pose of a manipulator end-effector. In many cases, when dynamics of human motion has to be passed to and reflected by the executive device (manipulator), the higher derivatives of the position signals are required. This signals can also be used in advanced centralized control laws with feedforwad compensation.

The methods for estimating the derivatives of a signal (up to second order) can be found in literature. In [6] velocities and accelerations are estimated for a single joint with a Kalman filter (KF). The authors shows that with increase of the sample rate the influence of the precise linear model of the joint becomes insignificant and it can be replace by simple consecutive integration scheme with the highest derivative as an input. This method is good for actuator with big reduction ratio (over 100) where the influence of the external torques is proportionally minimized and the scaling effect of the gear can be used to enhance the output shaft position measurements and the velocity/ acceleration estimation.

In [7] an approach to velocity estimation and filtering from an encoder measurements with M-method is proposed. In the core of the method is the variable (adaptive) noise covariance matrix of KF. The subsequent acceleration estimation is performed via a phase-locked-loop (PLL) structure. The review and analysis of other methods that use simplified system models or none at all for velocity estimation is given in [8]. All the method use advanced signal processing techniques mostly from frequency domain analysis.

The main problem with application of aforementioned methods to the device in this paper is dynamic and kinematic coupling in the parallel kinematics structure. The most of the methods show good results with actuators having big reduction ratio (100:1 and more) that result in significant decrease in the external torques influence and, as a result, simplification of the estimation procedure.

The work [9] describes a kinematic KF for a single joint. The filter use the fusion of the position sensor signal (encoder) and the acceleration estimation signal from an inertial sensor (MEMS). A similar approach can be found in [10] where the signals fusion of the positions on the joints and the accelerometers measurements of a biped robot is done through the kinematic model of the later and KF. The sensor fusion is not applicable for the haptic device in this paper since only positional measurements are available.

The authors of [11] utilize the extended KF with partial linearization of the dynamical model of a planar two DoF manipulator. The joints positions measurements and the accelerometer measurements placed at the end-effector are fused with KF to enhance the position estimation of the end-effector. In their subsequent work [12] a comparison between the extended KF and the particle filter for a two DoF manipulator with flexible joints is presented. The full dynamical model of the manipulator is used for simulation while simplified linear model is used for estimation. The state space vector contains the positions, velocities and acceleration of the joints along with their biases. 
The linear model itself is a simple discrete integrator. The study has shown the increase in the accuracy of the position measurement and the auxiliary acceleration estimation. In [13] presented a sliding mode filer (SMF) for estimation of time derivations of some continuous function. To avoid chattering phenomena or high-frequency oscillations signum function was replaced by hyperbolic tangent function resulting in saturated nonlinear smoothing filter.

The paper [14] contain filtering approach similar to KF based on interval analysis. The filter uses the system model and measurements to estimate the feasible state space vector interval given the intervals for the control and measurement noises. Unlike the classic KF this filter does not yield particular value of the state space vector but the interval it belongs to. While the classic KF give probabilistic estimate of the value this filter give guaranteed interval within which the value falls but not the value itself. It constitutes both strength and weakness of the filter since it can't be used directly in the control law without other means to specify a particular value within the interval.

In the present paper the estimation of the derivative of the position signals has several preconditions. First, only position measurements of the joints angles are available (sensor fusion is not possible). The dynamics of the parallel kinematic manipulator is intrinsically coupled that results in the limited performance of the linearization based approaches for the velocities and accelerations estimation. The absence of the gearbox in the actuators makes it impossible to consider the internal shafts of the motors as decoupled from external torques. Furthermore the enhancement of the accuracy of the velocity and acceleration estimation due to scaling effect is also unavailable. In the light of these preconditions the following approach is proposed. Consider the general dynamic model of the haptic device (1)

$$
\begin{aligned}
& B(q) \cdot \ddot{q}+C(q, \dot{q})+G(q)=\tau_{m}+\tau_{e x t} \\
& \tau_{m}=C_{m} \cdot i_{m} \\
& u_{m}=L_{m} \cdot \frac{d i_{m}}{d t}+R_{m} \cdot i_{m}+C_{e} \cdot \dot{q}
\end{aligned}
$$

Equation (1a) is the lagrangian representation of the general dynamic of the parallel and the series parts of the manipulator. In (1) $q, \dot{q}, \ddot{q} \in \mathbf{R}^{6}$ - joint positions and their derivative, $B(q)$-joint space inertia matrix, $C(q, \dot{q})-$ vector of the Coriolis and centrifugal torques, $G(q)$ - vector of gravity torques, $\tau_{m}$ - motor torques vector, $\tau_{\text {ext }}-$ external torques vector. Equation (1b) connects the motors currents and the motors torques where $C_{m}$ is the torque constant, $i_{m}$ - motors currents vector. The last equation (1c) is the dynamics of electromagnetic processes in the motor where $u_{m}-$ vector of motors voltages, $R_{m}, L_{m}$ - the diagonal matrices of the motors resistance and inductance, $C_{e}-$ the diagonal matrices of the motors electrical constants. If at the joint control level a fast enough current control loop (typically implemented as a PI regulator) (1b) and (1c) can be recuded to (1d)

$$
\tau_{m}=u_{m}^{\prime}
$$

where $u_{m}^{\prime}$ - vector of new control input that can be regarded as required troques.

Consider the extended state vector $Q=\left[q, \dot{q}, \tau_{e x t}^{\prime}\right]^{T}$. The equation (1a) can be written in state space form (2)

$$
\begin{aligned}
& \dot{Q}=A(q) \cdot Q+N(q) \cdot\left(\tau_{m}-G(q)-C(q, \dot{q})\right)+N(q) \cdot w \\
& y=Z \cdot Q+v
\end{aligned}
$$

Where

$$
\begin{gathered}
A=\left[\begin{array}{ccc}
0_{6 x 6} & I_{6 x 6} & 0_{6 x 6} \\
0_{6 x 6} & 0_{6 x 6} & B^{-1}(q) \\
0_{6 x 6} & 0_{6 x 6} & 0_{6 x 6}
\end{array}\right] \\
w=\tau_{e x t}
\end{gathered}
$$

$w \in \mathbf{R}^{n}, v \in \mathbf{R}^{n}$ - control and measurement noises presumably uncorrelated in wide sense, $\tau_{\text {ext }}^{\prime}-$ pseudostate giving the estimation of external torque (if $\tau_{\text {ext }}^{\prime}(0)=0$ this state does not affect the calculations).

For the KF derivation below following assumption have to be accepted. The joint positions and velocities $q, \dot{q}$ are constant within the time quantization interval. Thus, the matrix $A$ is assumed constant on the interval and its discretized version can be expressed through matrix exponent $A_{d k}=e^{A\left(t_{k}\right) \cdot T}$ where $T$ is the sample time. The discretized system then takes the form (3) 


$$
\begin{aligned}
& Q_{k+1}=A_{d k} \cdot Q_{k}+N_{d k} \cdot\left(\tau_{m k}-G\left(q_{k}\right)\right)+S_{k} \cdot w_{k} \\
& y_{k}=Z \cdot Q_{k}+v_{k}, \\
& Z \cdot Q_{k} \equiv q_{k} \\
& S_{k}=N_{d} \cdot B^{-1}\left(q_{k}\right)
\end{aligned}
$$

The observer for the system estimating the velocities and external torques is built with the minim error variance principle (KF) [15] as (4).

$$
\begin{aligned}
& K_{o b s k}=P_{k}^{-} \cdot Z^{T} \cdot\left[Z \cdot P_{k}^{-} \cdot Z^{T}+V_{k}\right]^{-1} \\
& \hat{Q}_{k}^{+}=\hat{Q}_{k}^{-}+K_{o b s k} \cdot\left[y_{k}-Z \cdot \hat{Q}_{k}^{-}\right] \\
& P_{k}^{+}=\left(I-K_{o b s k} \cdot Z\right) \cdot P_{k}^{-} \cdot\left(I-K_{o b s k} \cdot Z\right)^{-1}+K_{o b s k} \cdot V_{k} \cdot K_{o b s k}^{T} \\
& \hat{Q}_{k+1}^{-}=\hat{A}_{d} \cdot \hat{Q}_{k}^{+}+N_{d} \cdot B^{-1}\left(q_{k}\right) \cdot\left(\tau_{m k}-G\left(q_{k}\right)\right) \\
& P_{k+1}^{-}=\hat{A}_{d} \cdot P_{k}^{+} \cdot \hat{A}_{d k}^{T}+S_{k} \cdot W_{k} \cdot S_{k}^{T}
\end{aligned}
$$

where

$$
\hat{A}=\left[\begin{array}{ccc}
0_{6 x 6} & I_{6 x 6} & 0_{6 x 6} \\
0_{6 x 6} & 0_{6 x 6} & B^{-1}(q) \\
0_{6 x 6} & 0_{6 x 6} & 0_{6 x 6}
\end{array}\right],
$$

$\hat{A}_{d k}=e^{\hat{A}\left(q_{k}\right) \cdot T}, N_{d k}-$ corresponding matrices of the discrete model.

In (4) the supperscript "+" denoted the values after correction phase of KF, the supperscript "-" - respectively before correction phase (update). The subscript is the iteration number. Through $P_{k}^{*}$ error correlation matrix is denoted, $W_{k}, V_{k}$ are the correlation matrices of control $w_{k}$ and measurement $v_{k}$ noises (for simplicity taken constant but for some mode of operation can be functionally approximated), $K_{o b s k}$ - Kalman filer gain. In the model utilized for the presented KF the external torques $\tau_{\text {ext } k}$ are model as constant vector but other forms cab be found in publications. One such form is a stable dynamical system excited by white noise $w_{\tau}(5)$

$$
\tau_{\text {ext } k+1}=A_{\tau} \cdot \tau_{\text {ext } k}+w_{\tau k}
$$

The main problem of such model of external torques process is absence of the apriory knowledge about the external torques process and its parameters. For this reason $A_{\tau}$ is typically chosen in a from of a stable low pass filter (there is no recommendation, though, on the pole placement except for particular cases). The output of the filter (4) are the positions $\hat{q}_{k}$, velocities $\dot{\hat{q}}_{k}$ and external torques $\hat{\tau}_{\text {ext } k}$ estimates. The accelerations estimates are calculated by reordering (1a) as (6)

$$
\ddot{\hat{q}}_{k}=B^{-1}\left(\hat{q}_{k}\right) \cdot\left(\tau_{m k}+\hat{\tau}_{e x t k}-G\left(q_{k}\right)-C\left(q_{k}, \dot{\hat{q}}_{k}\right)\right)
$$

\section{Simulation results}

The parameters of the haptic device model are as follows. The radius of the base plate where the parallel mechanism actuators are place $R$ is taken $105 \mathrm{~mm}$, the radius of the joints placement on the moving plate $r$ is $54.5 \mathrm{~mm}$. The length of the link connected to where the parallel mechanism actuators (shoulder links) $l$ equals $100 \mathrm{~mm}$, the next links (elbow link) has length $\mathrm{L}$ of $200 \mathrm{~mm}$. The joints on the base plate (actuated) have numeration from 1 to 3 . The axes of the $4^{\text {th }}$ joint (series part of the mechanism) is placed at the center of the moving plate. The X-axes of the moving plate is directed upwards, the $\mathrm{Z}$-axes is perpendicular to the plate and toward the handle and a human operator. The pose of the $5^{\text {th }}$ joint from the center of the moving plate can be described by the vector $t_{5}=\left[\begin{array}{lll}0 & -0.1 & 0.1165\end{array}\right] \mathrm{mm}$. 
The pose of the $6^{\text {th }}$ joint with respect to the $5^{\text {th }}$ in initial angular positions (zeros by default) is $t_{6}=\left[\begin{array}{lll}-0.1 & 0.142 & 0\end{array}\right]$ $\mathrm{mm}$. The pose of the handle with respect to the 6th joint output shaft (in initial position) is $t_{h}=\left[\begin{array}{lll}0.1 & 0 & 0\end{array}\right] \mathrm{mm}$. The mass of the moving platform $m_{p}=0.1 \mathrm{~kg}$. The masses of the shoulder and elbow links are taken equal $m_{1}=m_{2}=0.05 \mathrm{~kg}$. The masses of the orientational DoFs links are also taken equal $m_{4}=m_{5}=0.015 \mathrm{~kg}$. For the purposes of the simulation the motors inertia is also taken equal for all joints $J_{m 1}=1 \cdot 10^{-5} \mathrm{~kg} \cdot \mathrm{m}^{2}$. The inertia tensors of the orientational DoFs links are also taken as equal diagonal matrices with elements $I_{x x}=I_{y y}=I_{z z}=1 \cdot 10^{-3} \mathrm{~kg} \cdot \mathrm{m}^{2}$. The positions of the mass centers are taken in the centers of the links: $r_{c g 5}=\left[\begin{array}{llll}0 & -0.1 & 0.1165\end{array}\right] / 2 \mathrm{~m}, \mathrm{r}_{c g 6}=\left[\begin{array}{lllll}-0.1 & 0.142 & 0\end{array}\right] / 2 \mathrm{~m}, r_{c g h}=\left[\begin{array}{lll}0.1 & 0 & 0\end{array}\right] / 2$ $\mathrm{m}$. The system was excited by the external force/torque at the handle (7)

$$
\begin{aligned}
& f_{x}=10 \cdot \sin (5 \cdot t) \cdot \cos (3 \cdot t) \\
& f_{y}=3 \cdot \sin (10 \cdot t) \\
& f_{z}=4 \cdot \cos (t)
\end{aligned}
$$

$$
\begin{aligned}
\tau_{x} & =1.1 \cdot \sin (7 \cdot t) \\
\tau_{y} & =2.4 \cdot \cos (4 \cdot t) \\
\tau_{z} & =2
\end{aligned}
$$

The measurement noise is taken as random Gaussian noise independent for all joints with the standard deviation $\sigma=0.1$. The KF control $Q$ and measurement $R$ correlation matrices are taken diagonal with equal elements with value 0,0001 and 0.01 respectively. The sample time is take $0,001 \mathrm{sec}$. that is typical for the centralized control loops found in literature (especially for force/torque controlled systems). The simulation results for a single joint are presented in fig. 6.
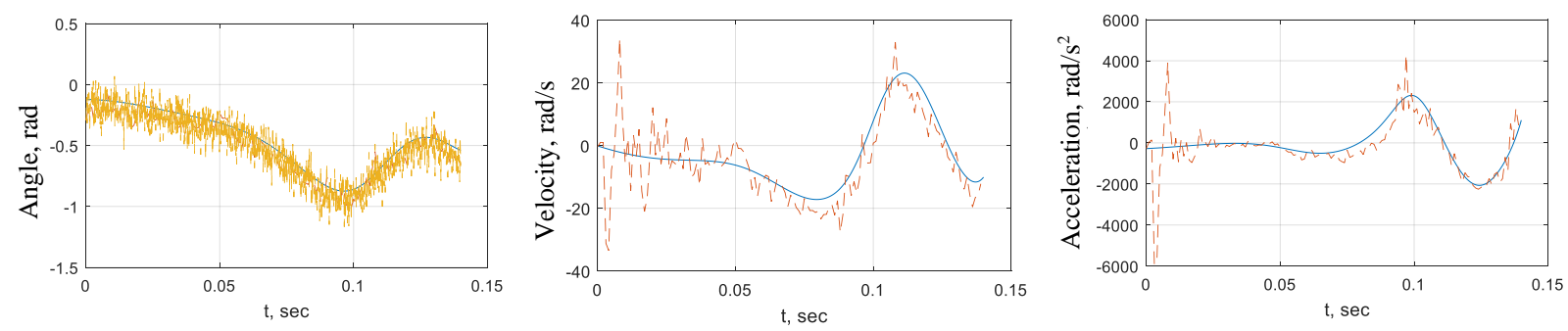

Fig. 6. Simulation results for a single joint: left to right is position, velocity acceleration (bold line is the original signal (no noise), dashed line is the reconstructed signal)

It can be seen from figure 6 that the filter reconstructs the acceleration with insignificant phase delay. In steady state the MSE for position is $0,09 \mathrm{rad}$, for velocity - $10 \mathrm{rad} / \mathrm{s}$. Absolute MSE for acceleration is $500 \mathrm{rad} / \mathrm{s}^{2}$ in steady state (relatively less that $20 \%$ of the magnitude).

In order to estimate the possible quality of velocity/acceleration estimation the matrices $Q$ and $R$ were varied in series of experiments (from their optimal value). Qualitatively the results of this experiments can be summarized as follows. For relatively large values of Q and/or R (bigger that that corresponding to the noises) the filter gives good results for position estimation but exhibits significant phase delays for velocities and accelerations estimation. For some critical values this resulted in high peaks and oscillations when the actual velocities/acceleration change very fast (faster than the filter bandwidth). This effect is mainly due to non-linear dependences of the dynamic model from them (figure 7). The same effect was observed when the filtered positions $\left(\hat{q}_{k}\right)$ instead of measured $\left(q_{k}\right)$ was used in dynamic model for torques values estimation. On the other hand setting the correlation matrices coefficients too small resulted in the insufficient filtering and unreasonably noisy acceleration estimation while the mean value of the filtered signal comes closer to the actual values (figure 8).
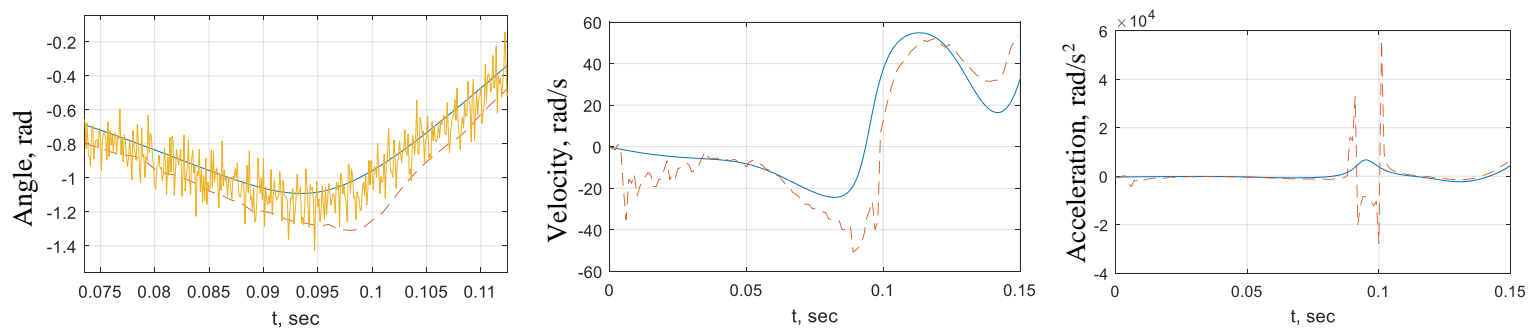

Fig. 7. The nonlinear effect of lowering the bandwidth of the filter by choosing big values in correlation matrices and/or using filtered position/velocity values in the dynamic model (bold line is the original signal (bold line is the original signal (no noise), dashed line is the reconstructed signal) 

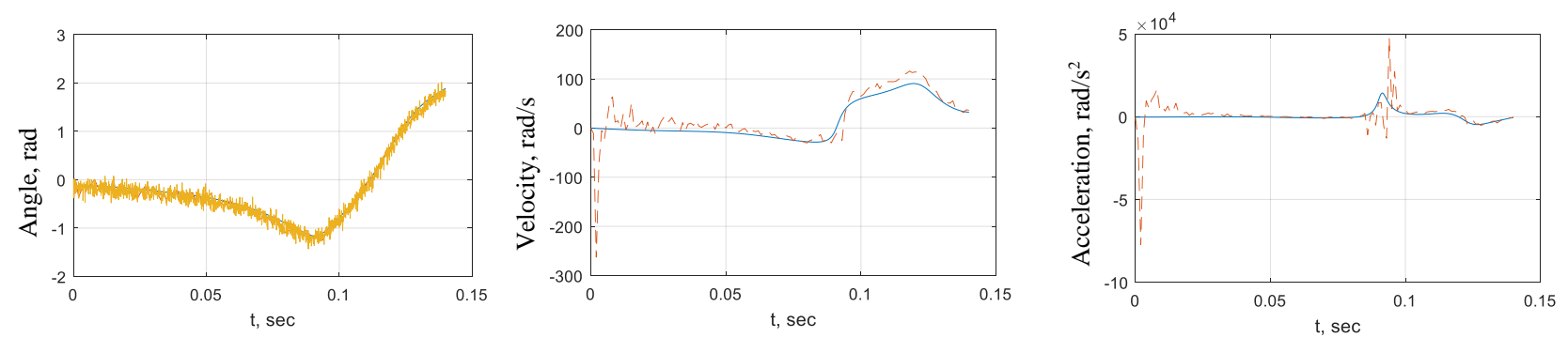

Fig. 8. The nonlinear effect of increasing ring the bandwidth of the filter by choosing small values in correlation matrices (bold line is the original signal (bold line is the original signal (no noise), dashed line is the reconstructed signal)

Thus the proposed approach shows certain limitation imposed by the filter bandwidth and nonlinear components of the dynamic model. The noise magnitude is also a major factor reducing the accuracy of acceleration measurements.

\section{Discussions}

The simulation has shown the validity of the presented approach for the acceleration estimation for the parallelserial mechanisms. The main fiture of the approach in this paper from the mentioned above is utilizing of full dynamic model in filter construction without direct linealization (no derivatives of non-linear functions for linearization purposes implied) that makes it different from the classical exteneded KF typically found in literature. The acceleration estimation is done by direct calculation using the values of the velocities and external torques estimated by the KF.

One and probably the main disadvantage of the proposed approach is its computational complexity. Other approaches considered above utilize some sort of simplified models that significantly fasten the calculations. In particular [12] describes the system model for acceleration measurements as a three-stage discrete integrators with zero-order-hold (ZOH). The state vector is chosen as $\breve{Q}_{k}=\left[q_{k}, \dot{q}_{k}, \ddot{q}_{k}, b_{\ddot{q} k}\right]^{T} \in \mathbf{R}^{6 \cdot 4}$ (the dimensions corresponds to the task in the present paper). The first three components of the state vector $\breve{q}, \dot{\vec{q}}, \ddot{\vec{q}}$ are the corresponding estimates of the positions, velocities and accelerations while $b_{\ddot{q}}$ are estimated biases for positions and accelerations (this are the unavoidable error accumulator which role in the proposed algorithm plays $\left.\tau_{e x t}^{\prime}\right)$. Thus the discrete transition matrix for the system in [12] takes the form (8)

$$
F=\left[\begin{array}{cccc}
I_{6 x 6} & I_{6 x 6} \cdot T & \frac{I_{6 x 6} \cdot T^{2}}{2} & 0_{6 x 6} \\
0_{6 x 6} & I_{6 x 6} & I_{6 x 6} \cdot T & 0_{6 x 6} \\
0_{6 x 6} & 0_{6 x 6} & I_{6 x 6} & I_{6 x 6} \\
0_{6 x 6} & 0_{6 x 6} & 0_{6 x 6} & I_{6 x 6}
\end{array}\right]
$$

where $T$ is the sample time.

The fundamental disadvantage of this approach (and the ones similar to it) is too small coefficients in transition matrix for the acceleration terms $I_{6 \times 6} \cdot T^{2} / 2$ that leads to poor accuracy of the estimation. This coefficients are decreasing with sample time that makes the essence of contradiction when fast processes are to be processed. The error accumulators can also introduce the systematic error into the acceleration estimates (while improving that of the positions and velocities). The undoubted advantage of the method is computational efficiency.

The approach in [7] includes PLL to estimate the acceleration from the velocity in turn estimated by KF. The system model is still the double integrator. As the authors themselves mention the method gives poor results for small velocities and accelerations but is yet batter than direct differentiating. The velocities and acceleration estimates are especially bad when the noise magnitude dominates that of the positional signal. While the proposed method suffers the same problem but gives better results than that in [12]. Comparison with [7] has not been performed yet.

All the linear model that do not use nonlinear terms in torques (velocities) equations and applied for the task of derivatives estimation will have inferior accuracy in comparison to nonlinear ones because the former does not utilize the local properties of the manifold the dynamical system lives on.

One of the possible ways to improve the situation is to introduce one more derivative order (jerk) into the state model. This brings up the integration action for the acceleration estimation but requires for the torque equation differentiation that is both analytically and computationally difficult. Furthermore the sample time problem discussed above will arise. 
Another major problem is limitation of the bandwidth due to the noise magnitude. The bandwidth is always the outcome of the balancing between the filtering and MSE of derivatives estimations. The optimal filter tuning might exist for this problem which is the topic of the future research.

Another direction of the future research is application of cascades of filters that sequentially enhance the accuracy. This approach may be fruitful if nonlinear filtering techniques (like neural networks or adaptive filters) are applied. On the way of increasing the computational efficiency simplification of the dynamic model can give some benefits. Neglecting some links inertias and masses may help to reduce several terms in equations but accuracy need to be checked in the process. These and other aspects will be elaborated in the future as well as the implementation on the target hardware where additionally the influence of fixed point calculations is to be considered.

\section{Conclusion}

The design of the 7 DoF haptic device is presented in the paper. The workspace and the accuracy maps of the mechanism are given. Particular attention is paid to the signal processing that includes position signals filtering as well as velocity and acceleration estimation. Nonlinear system observer based on the Kalman filter is proposed for accelerations and velocities estimation. The estimation has shown to be non-trivial one and no universal technical solution has been found in literature. The performance of the proposed observer is evaluated and discussed along with the directions of the further improvements. Testing on the designated hardware is yet to be done.

\section{Acknowledgments}

The Applied scientific investigations are carried out with the financial support of the Russian Federation represented by the Russian Ministry of Education and Science. The unique identifier of the Applied scientific investigations is 9.13275.2018/12.1.

\section{References}

[1] Petrov B.A., Andreenko S.M., Voroshilov M.S. (1975). Proektirovanie privodov manipulyatorov [Design of the manipulator drives]. Leningrad, Mashinostroenie Publ., 312 p.

[2] Kraft Robotic Science and Technology [Electronic Source] / Free Access free.-Screen title: www.krafttelerobotics.com.

[3] Titov V., Shardyko I., Isaenko S.(2014). Force-Torque Control Implementation for 2 DoF Manipulator//24th DAAAM International Symposium on Intelligent Manufacturing and Automation, Procedia Engineering 69, 2013, - pp. 1232-1241.

[4] Haptic Devices Based on Parallel Mechanisms [Electronic Source] / Free Access free.-Screen title: https://www.parallemic.org/Reviews/Review003.html.

[5] A.Tobergte, P. Helmer, U. Hagn etc. (2011). The sigma.7 haptic interface for MiroSurge: A new bi-manual surgical console In International Conference on Intelligent Robots and Systems, San Francisco, CA, USA.

[6] Belanger P.R., Dobrovolny P., Helmy A., Zhang X. (1998). Estimation of Angular Velocity and Acceleration from Shaft-Encoder Measurements, The International Journal of Robotics Research Vol. 17, No. 11, November, pp. 1225 1233.

[7] Wang S, Wan S. (1998). Velocity and acceleration computations by single-dimensional Kalman filter with adaptive noise variance. Elektrotechniczny. 5: 283-287.

[8] L. Bascetta and G. Magnani. (2009). "Velocity Estimation: Assessing the Performance of Non-Model-Based Techniques," IEEE Transactions on Control Systems Technology, vol. 17, no. 2, pp. 424-434.

[9] Jeon S. (2010). State Estimation Based on Kinematic Models Considering Characteristics of Sensors, 2010 American Control Conference Marriott Waterfront, Baltimore, MD, USA June 30-July 02.

[10] Wawrzynsk P., Mozaryn J.' and Klimaszewski J. (2013). Robust velocity estimation for legged robot using on-board sensors data fusion, in 2013 18th International Conference on Methods and Models in Automation and Robotics (MMAR), 26-29 Aug., DOI: 10.1109/MMAR.2013.6670000.

[11] P. Axelsson, M. Norrlöf, E. Wernholt, and F. Gustafsson, (2010). "Extended kalman filter applied to industrial manipulators," in Proceedings of Reglermöte 2010, Lund, Sweden.

[12] Axelsson P., Karlsson R. and Norrlöf M. (2012). Bayesian State Estimation of a Flexible Industrial Robot, Control Engineering Practice, (20), 11, 1220-1228. [URL:http://dx.doi.org/10.1016/j.conengprac.2012.06.004].

[13] Stevanovic, S[tojan]; Kasac, J[osip] \& Stepanic, J[osip] (2012). Robust Tracking Control of a Quadrotor Helicopter Without Velocity Measurement, Annals of DAAAM for 2012 \& Proceedings of the 23rd International DAAAM Symposium, Volume 23, No.1, ISSN 2304-1382, ISBN 978-3-901509-91-9;

[14] Zbranek, P[avel] \& Vesely, L[ibor] (2010). Interval Analysis in Nonlinear State Estimation, Annals of DAAAM for 2010 \& Proceedings of the 21 st International DAAAM Symposium, Volume 21, No. 1, ISSN 1726-9679 ISBN 978 3-901509-73-5;

[15] Chui C.K., Chen G. (2009). Kalman Filtering with Real-Time Applications (4th ed.), Springer-Verlag Berlin Heidelberg, DOI 10.1007/978-3-540-87849-0. 